\title{
Spatial data sharing implementation in Malaysia's marine organisations: a case study
}

\begin{abstract}
This paper discusses the explanatory study of spatial information sharing in Malaysian marine-related organisations. A semi-structured interview is used to explore the GIS implementation in the organisation. This paper also examines the drivers and issues in implementing marine spatial data sharing within and between marine organisations. Eleven marine and coastal-related organisations were interviewed. Thematic and SWOT analysis was selected to categorise information into several themes and factors including internal and external factors that affected the GIS and spatial data sharing implementation in the organisations. The results revealed that most of the respondents were aware of the importance of GIS and spatial data sharing to support certain aspects of the management of the organisation. The results also show the lack of planning and implementation of GIS, lack of awareness of the importance of GIS and spatial data sharing, and lack of communication and cooperation within and between organisations. To improve the marine spatial information sharing, two strategies was proposed: improving GIS strategic planning to focus on GIS planning and development in the organisation, and enhancing cooperation to focus on developing the connection and communication between organisations to facilitate spatial information sharing.
\end{abstract}

Keyword: Marine organisational cooperation; Marine spatial data infrastructure; Marine spatial data sharing 